\title{
Adsorption of Hexacontane on Hexagonal Boron Nitride
}

\author{
Juan D. Albar, ${ }^{\dagger}$ Vladimir V. Korolkov, ${ }^{\dagger}$ Matteo Baldoni, ${ }^{\ddagger}, \S$ Kenji Watanabe, ${ }^{\circ}$ Takashi Taniguchi, ${ }^{\|}$ \\ Elena Besley, ${ }^{\ddagger}$ and Peter H. Beton* ${ }^{*} \dagger$ (1) \\ ${ }^{\dagger}$ School of Physics and Astronomy and ${ }^{\ddagger}$ School of Chemistry, University of Nottingham, Nottingham NG7 2RD, U.K. \\ ${ }^{\S}$ Istituto per lo Studio dei Materiali Nanostrutturati (ISMN), Consiglio Nazionale delle Ricerche (CNR), Via P. Gobetti 101, \\ Bologna 40129, Italy \\ "National Institute for Materials Science, 1-1 Namiki, Tsukuba, Ibaraki 305-0044, Japan
}

Supporting Information

\begin{abstract}
We have investigated the adsorption of hexacontane $\left(\mathrm{C}_{60} \mathrm{H}_{122}\right)$ on hexagonal boron nitride $(\mathrm{hBN})$ using atomic force microscopy (AFM). The molecules can be deposited either by sublimation or from solution and form lamellar rows with dimensions of the order of $0.1-1 \mu \mathrm{m}$ in three different rotational domains. High-resolution AFM images reveal that, similar to alkanes on graphite, the molecules are adsorbed parallel to the lattice vectors of $\mathrm{hBN}$ and we show using molecular mechanics that this corresponds to the lowest energy configuration. Lamellar rows with the same periodicity are observed even when several layers of
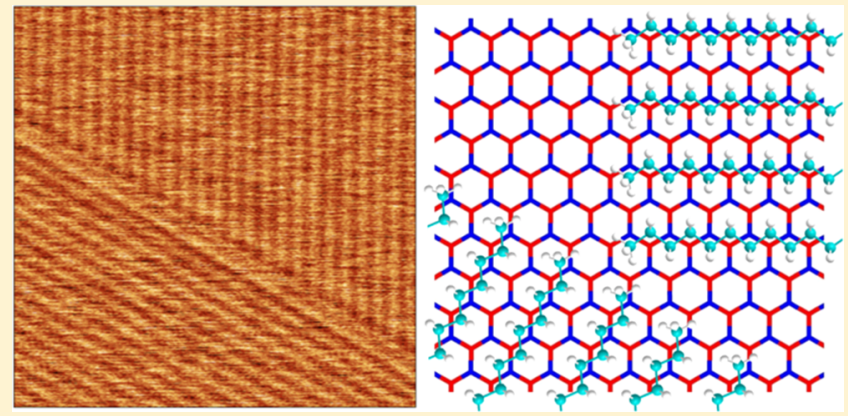
hexacontane are deposited, although there is some orientational disorder in these multilayers. We also observe heat-induced modification of hexacontane, including recrystallization. We compare our results with recent $\mathrm{X}$-ray studies of alkane adsorption on $\mathrm{hBN}$ and discuss the possible role of alkanes on steering molecular self-assembly on hBN.
\end{abstract}

\section{INTRODUCTION}

Over the past few decades, there has been a continuing interest in the adsorption of alkanes on surfaces, since they play important roles in catalysis, ${ }^{1,2}$ lubrication, ${ }^{3}$ and passivation. ${ }^{4}$ The adsorption of these readily available, low-cost, and nontoxic materials on various surfaces has been studied in depth, particularly on metals ${ }^{5-7}$ and graphite, ${ }^{8-15}$ where they provide a route to promote complex organization through twodimensional supramolecular assembly. ${ }^{16-20}$ In parallel, the recent surge in activities in two-dimensional materials ${ }^{21}$ has led to a renewed interest in hexagonal boron nitride ( $h B N)$, a structural isomorph of graphite. This layered material can serve as an atomically flat tunneling barrier, ${ }^{22}$ is mostly free of charge impurities, ${ }^{23}$ and when used as a substrate for graphene, greatly improves electrical performance. ${ }^{24}$ It has also been shown that $\mathrm{hBN}$, both in the form of monolayers (MLs) grown on metals $^{25-28}$ and thicker exfoliated flakes, ${ }^{29}$ can support the formation of two-dimensional supramolecular assemblies. Furthermore, hBN has been used successfully as a dielectric in organic transistors. ${ }^{30,31}$ However, the adsorption of alkanes on hBN has so far been studied only using diffraction techniques, ${ }^{32,33}$ with no direct determination of their real-space arrangement and potential to control supramolecular organization.

In this paper, we address this question through an investigation of the adsorption of a long-chain alkane, hexacontane $\left(\mathrm{C}_{60} \mathrm{H}_{122}\right)$, on $\mathrm{hBN}$. The alkane can be deposited either by sublimation or from solution. We find, using atomic force microscopy (AFM), that the alkanes lay flat on the surface with the molecular axis parallel the zigzag direction of $\mathrm{hBN}$, forming lamellar rows, similar to those formed by longchain alkanes deposited on graphite. Upon deposition of more than one layer of hexacontane, higher layers are formed that propagate the row geometry but these are more disordered and weakly bound and are thus more susceptible to heat- and tipinduced modifications.

\section{EXPERIMENTAL SECTION}

Hexacontane was purchased from Sigma-Aldrich ( $\geq 99.8 \%$ ) and used without further purification. Following our previous work, ${ }^{29,34,35}$ flakes of hBN with typical lateral dimensions of $\sim 20-50 \mu \mathrm{m}$ and thicknesses $10-100 \mathrm{~nm}$ were exfoliated from high-temperature- and high-pressure-grown bulk hBN crystals, ${ }^{36}$ transferred onto $5 \times 5 \mathrm{~mm}^{2} \mathrm{Si} / \mathrm{SiO}_{2}$ wafers, and then cleaned by immersion in toluene (99.9\% Chromasolv for high performance liquid chromatography, Sigma-Aldrich) overnight, followed by heating at $400{ }^{\circ} \mathrm{C}$ for $8 \mathrm{~h}$ in a flow of $0.15 \mathrm{sL} / \mathrm{min}$ of $\mathrm{Ar} / \mathrm{H}_{2}$ (95:5). In addition, samples were flame-annealed immediately before hexacontane deposition. Hexacontane was chosen since it can be deposited either from solution or by

Received: October 18, 2018

Revised: November 13, 2018

Published: November 13, 2018 
sublimation from a crucible in a Knudsen cell resistively heated to $200{ }^{\circ} \mathrm{C}$ (this temperature is significantly higher than that for many smaller available alkanes, thus minimizing the possibility of long-term contamination of the vacuum chamber). The pressure during sublimation was $10^{-7}$ Torr. No heating was applied to the sample during the deposition process. The layer thickness was determined using a quartz crystal thickness monitor, which was calibrated from the surface coverage of the dendritic structures formed by hexacontane on the $\mathrm{SiO}_{2}$ surface. $^{37}$ In the case of solution deposition, the sample was immersed for $1 \mathrm{~min}$ in a solution of hexacontane in toluene $(10 \mu \mathrm{g} / \mathrm{mL})$ under standard conditions. AFM images were acquired using an Asylum Research Cypher-S instrument under standard conditions in conjunction with Multi75AI-G cantilevers (Budget Sensors). All images were obtained in AC (tapping) mode unless stated otherwise.

\section{RESULTS AND DISCUSSION}

An AFM image of the surface of an hBN flake acquired after the deposition, by sublimation, of 1 monolayer (ML) of hexacontane is shown in Figure 1. As revealed by a higher

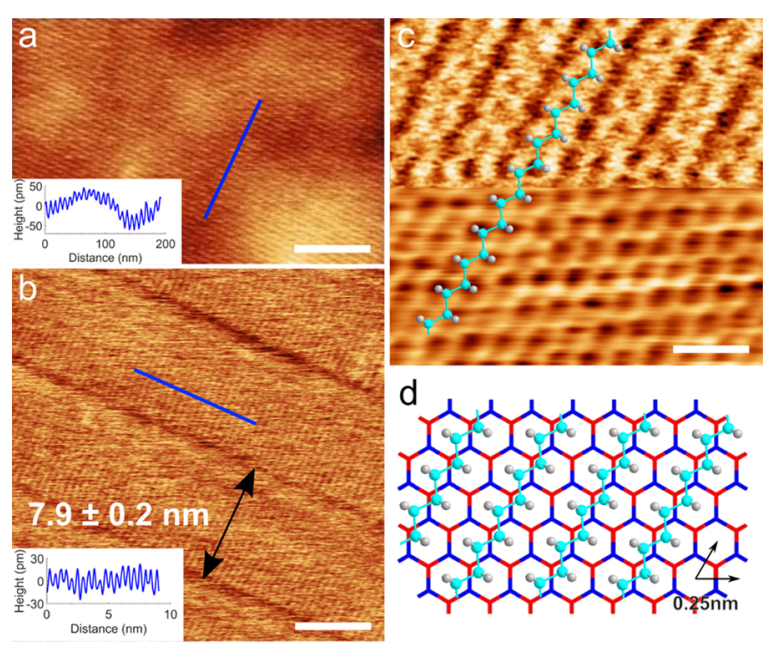

Figure 1. AFM images of $1 \mathrm{ML}$ of hexacontane sublimed onto hBN. (a) Large area image of hexacontane lamellar rows; inset: height profile along the trajectory marked in the image; (b) high-resolution topography image showing the molecular arrangement within the lamellae; inset: height profile along the trajectory marked in image; (c) high-resolution AFM images showing individual hexacontane molecules on the $\mathrm{hBN}$ lattice (top half) and the bare hBN lattice on the same area after molecules were removed (bottom half). The top half corresponds to a topography image, whereas the bottom one corresponds to a frequency modulation image. (d) Schematic showing the registry of hexacontane with respect to the hBN lattice. The arrows represent the lattice vectors. Scale bars: (a) $100 \mathrm{~nm}$, (b) $5 \mathrm{~nm}$, and (c) $1 \mathrm{~nm}$.

magnification image on Figure $1 b$, we observe a series of rows running across the surface and a height profile (inset) along a line perpendicular to them shows that their amplitude is $20 \mathrm{pm}$ and their separation (determined from a Fourier transform) is $7.9 \pm 0.2 \mathrm{~nm}$. Additional irregular contrast variations are observed due to the roughness of the underlying $\mathrm{SiO}_{2}$ substrate on which the hBN flake is mounted. These background fluctuations have a peak-to-peak height variation of $50 \mathrm{pm}$ and a characteristic length scale of $\sim 100 \mathrm{~nm}$ and are not associated with the molecules.
The lines are very similar to those observed when alkanes adsorb on graphite and form lamellar rows in which the linear molecules are stacked perpendicular to the row axis. A higher magnification AFM image in Figure $1 \mathrm{~b}$ shows that the rows on $\mathrm{hBN}$ correspond to similar lamellae in which the individual molecules can be resolved and, as expected, are perpendicular to the row direction. The distance between molecules in the same row is $0.44 \pm 0.02 \mathrm{~nm}$, very close to the value reported for alkanes on graphite. The inset shows a profile taken across 20 molecules, as indicated by the path in blue. We typically observe domains with area $1-5 \mu \mathrm{m}^{2}$ in one of the three orientations, with an angular separation of $120^{\circ}$ (see below).

To determine the orientation of the molecules with respect to the underlying $\mathrm{hBN}$ substrate, we employ a protocol that we have previously used in the study of supramolecular heterostructures and the properties of adsorbed molecules on black phosphorus. ${ }^{38}$ By increasing the setpoint used in AFM imaging, it is possible to partially remove the alkane chains, allowing the local imaging of the underlying substrate. Figure 1c shows an overlay in which the top segment corresponds to the upper half of an image acquired at a low setpoint whereas the bottom segment is the lower half of an image of the same area acquired at a higher setpoint (the complete images acquired at high and low setpoint are included in the Supporting Information (SI)). The hBN surface shown in Figure $1 \mathrm{c}$ has a coverage of $1 \mathrm{ML}$, and the molecular chains can be identified in the upper half of the composite running from bottom-left to top-right. The bottom half of Figure 1c shows the exposed hBN lattice, which allows the identification of the orientation of the lattice vectors. A comparison between the images shows that the molecules run parallel to the lattice vector (the zigzag direction) of $\mathrm{hBN}$, as shown schematically in the structural diagram in Figure 1d.

The arrangement in Figure 1d is consistent with a model derived from X-ray studies of alkanes deposited on hBN by Arnold et al. $^{32}$ who find two large-scale morphologies, including a rowlike phase for long-chain alkanes, which is in agreement with our results. Although the precise registry of the molecules with the lattice cannot be determined from our images, the results in Figure 1 are consistent with the placement of $\mathrm{CH}_{2}$ groups on top of the hexagon centers within the hBN lattice, similar to the widely accepted model proposed by Groszek for linear alkanes on graphite. ${ }^{39}$ The spacing of neighboring molecules is consistent with that calculated $^{9}$ for alkanes on graphite lying with their carbon backbone parallel, ${ }^{14}$ rather than perpendicular ${ }^{13}$ to the surface. The topographic detail in Figure 1c is also consistent with this conformation; we see a bright topographic feature in the position attributed to each carbon atom, rather than alternate atoms that would be expected for the perpendicular configuration.

We have investigated the energetics of alkane adsorption on hBN using molecular mechanics employing LAMMPS simulation package ${ }^{40}$ in combination with the optimized potential for liquid solutions, ${ }^{41}$ optimized potential for liquid simulations potential, which was optimized for long-chain hydrocarbons. The hBN substrate was treated as a rigid monolayer with atoms fixed at their crystallographic positions. The Lennard-Jones parameters were taken from Lee. ${ }^{42}$ More details are provided in the SI. In particular, we have calculated the dependence of the adsorption energy of a single alkane chain on the relative orientation of the molecular axis and the hBN lattice. This has been evaluated through a lateral slide 
(a)

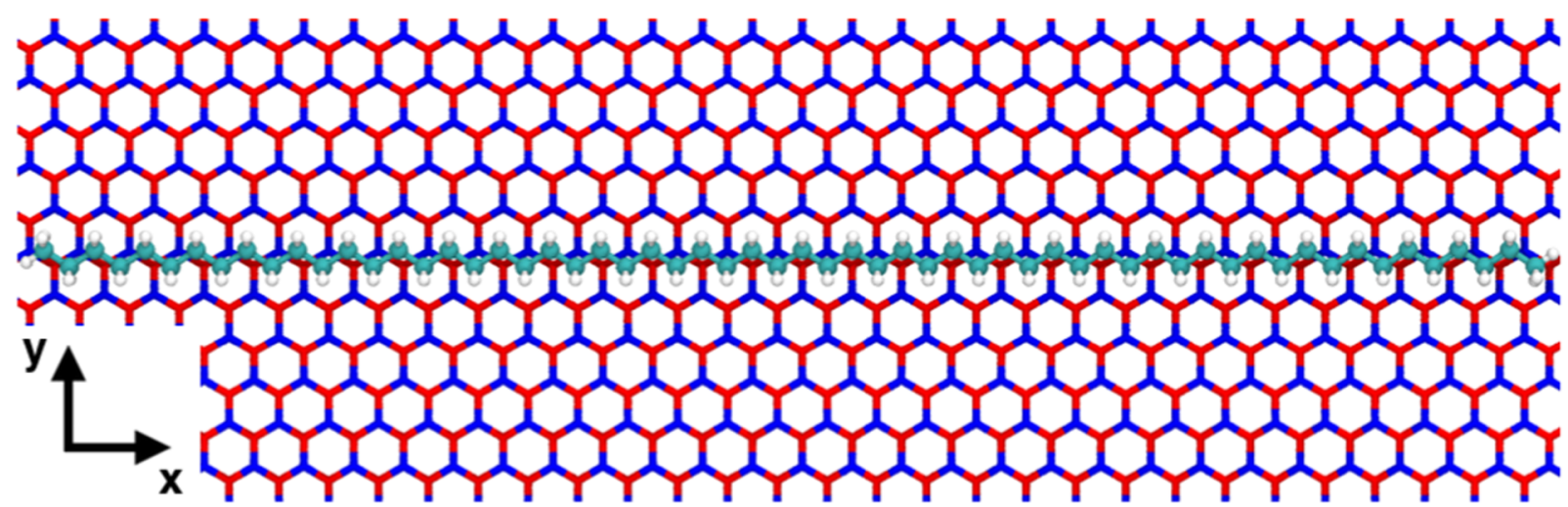

(b)

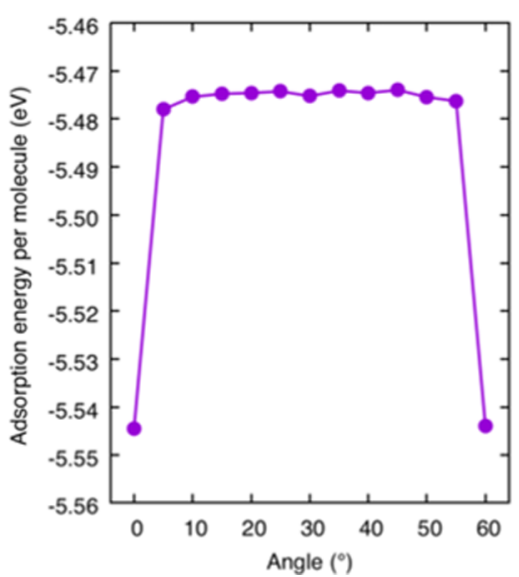

(c)

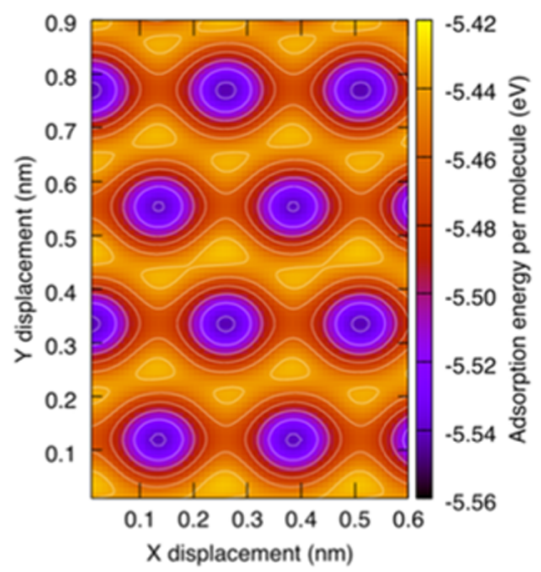

(d)

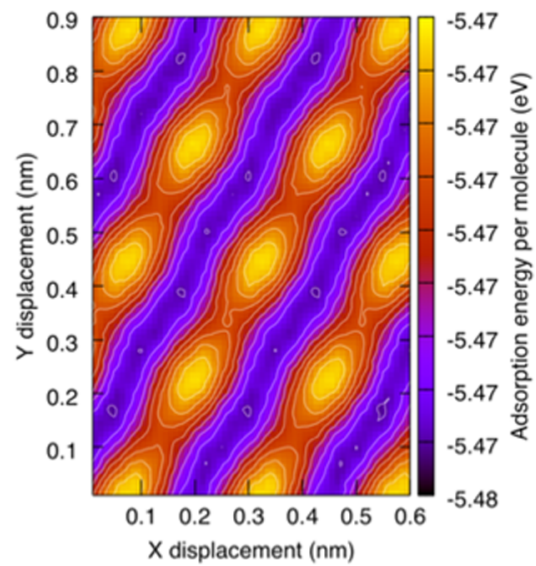

Figure 2. (a) Optimized structure of a hexacontane molecule on hBN surface; (b) adsorption energy as a function of the angle between the principal axis of the hexacontane molecule and the zigzag direction of the hBN lattice; and (c) potential energy surface at $0^{\circ}$ and (d) $20^{\circ}$ rotation.

analysis of the potential energy surface (PES) for different orientations of the adsorbed molecule (see Figure 2). A single hexacontane molecule was initially optimized in vacuum; the calculated $\mathrm{C}-\mathrm{C}-\mathrm{C}$ spacing along the chain is $0.255 \mathrm{~nm}$, close to the value taken for the lattice constant of hBN, $0.250 \mathrm{~nm}$. The optimized hexacontane molecule was then placed at a height $0.35 \mathrm{~nm}$ above the model hBN surface, with the plane of the carbon atoms parallel to the substrate. The molecule was next moved as a rigid body along the $x$ and $y$ axes (defined in Figure 2a) in steps of $0.01 \mathrm{~nm}$, as in our previous work. ${ }^{35}$ At each step, the adsorption energy was calculated as $E\left(\mathrm{C}_{60} \mathrm{H}_{122} @\right.$ $\mathrm{hBN})-E\left(\mathrm{C}_{60} \mathrm{H}_{122}\right)-E(\mathrm{hBN})$.

Our calculations show that, in agreement with experiment, the lowest energy corresponds to orientations where a hexacontane molecule is aligned with the $\mathrm{hBN}$ zigzag direction (Figure 2a); the adsorption energy in this configuration is favored by approximately $0.07 \mathrm{eV} /$ molecule (Figure $2 \mathrm{~b}$ ). The energies in Figure $2 \mathrm{~b}$ correspond to the minimum energies extracted from the PES profiles for each angle of rotation. Examples of the PES profile for alignment angles of 0 and $20^{\circ}$ are shown in Figure 2c,d. After the full relaxation of $\mathrm{C}_{60} \mathrm{H}_{122}$ molecule on hBN, the adsorption energy at $0^{\circ}$ is $-5.93 \mathrm{eV}$ (the actual structure is shown in Figure 2a). The results have also been confirmed for multiple (eight) alkane chains adsorbed on the $\mathrm{hBN}$ surface; after the minimization of the total energy, all eight molecules are positioned in the same preferential adsorption sites, as in the case of a single chain, giving an intermolecular distance of $0.435 \mathrm{~nm}$ corresponding to a commensurate arrangement on the hBN layer. The interaction energy between the chains have been estimated as $-0.13 \mathrm{eV}$ per pair. The spacing and orientation of the alkanes are in excellent agreement with our experimental observations.

Figure 3 shows images of hBN on which layers of hexacontane with different thicknesses have been sublimed. For sub-monolayer coverages (Figure 3a,b), isolated islands are formed with typical lateral sizes of $\sim 1 \mu \mathrm{m}$ and a height that is comparable to the roughness of the hBN flake. The island at the center of Figure $3 \mathrm{a}$ is shown at higher magnification (area highlighted in Figure $3 a$ ) in Figure $3 b$, and from the accompanying height profile (along the red line), we determine a height of $0.4 \pm 0.1 \mathrm{~nm}$ consistent with a partial monolayer of flat lying molecules. The direction of the lamellar rows is identified by the arrow; the rows run in a common direction for a given island, indicating a single extended domain of molecular ordering. An even higher magnification image (area highlighted in Figure $3 b$ ) is shown in Figure $3 c$ where the lamellar rows are resolved. The isolated hexacontane islands are rather unstable, and after acquiring several images, their shape can be modified or they can be removed completely; this tip-molecule interaction also accounts for the distortion in the lamellar rows, which is prevalent during small-scale scans, especially at sub-monolayer coverages. 


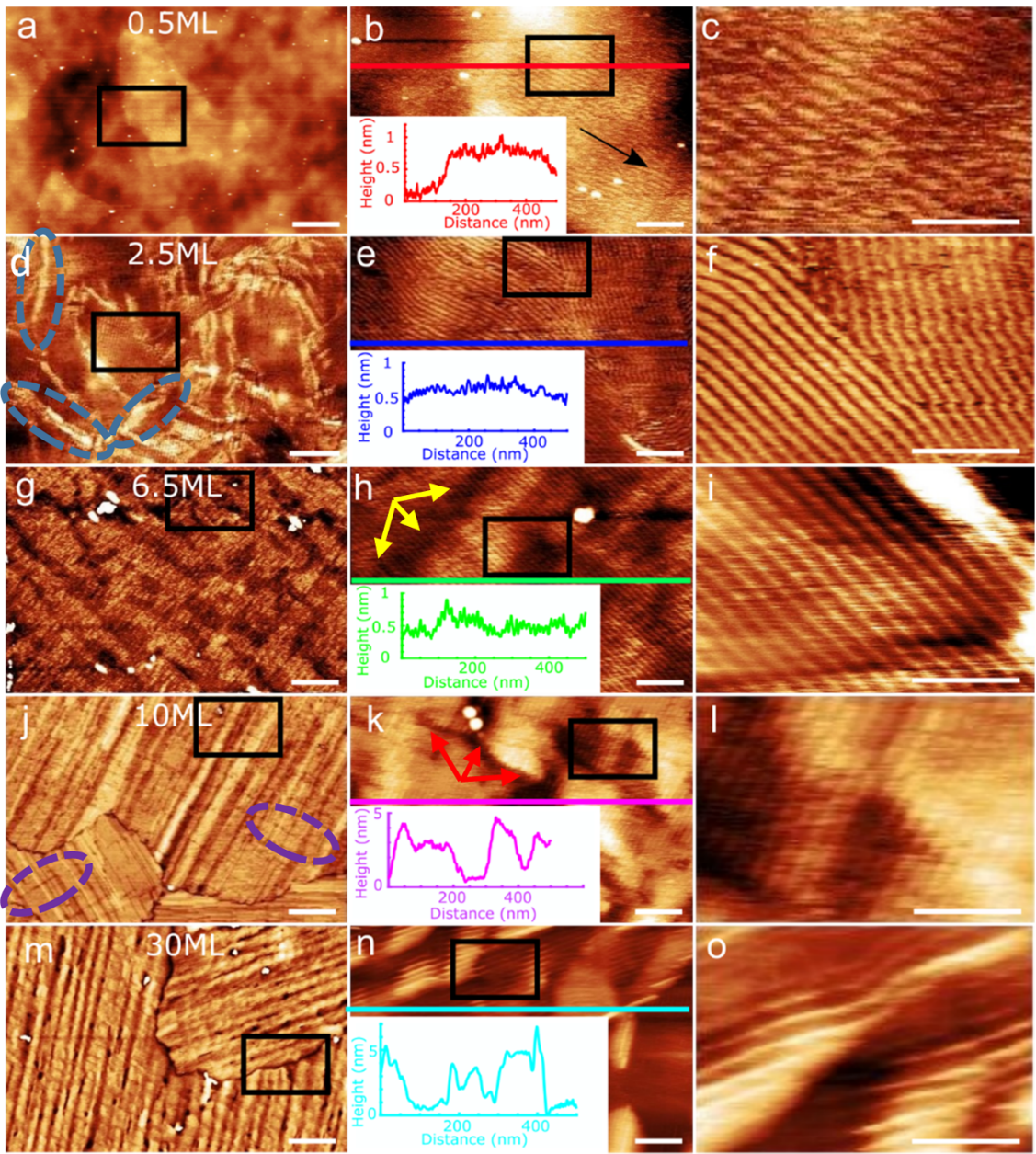

Figure 3. AFM images for different thicknesses of hexacontane. (a-c) $0.5 \mathrm{ML},(\mathrm{d}-\mathrm{f}) 2.5 \mathrm{ML},(\mathrm{g}-\mathrm{i}) 6.5 \mathrm{ML}$ (j-1) $10 \mathrm{ML}$, and (m-o) $30 \mathrm{ML}$. The boxes highlighted in black on the images in the left and center columns show the areas where the zoomed images in, respectively, the center and right columns were acquired. In the center column, profiles running along the paths indicated in different colors are overlaid on each image. The right column shows images in which the lamellar rows can be resolved for each thickness. Scale bars: left column, $400 \mathrm{~nm}$; middle column, $100 \mathrm{~nm}$; right column, $50 \mathrm{~nm}$.

At a coverage of $2.5 \mathrm{ML}$, topographically flat regions coexist with elongated features with higher apparent brightness (Figure $3 \mathrm{~d}-\mathrm{f}$ ); see features highlighted by dashed blue ellipses in Figure $3 \mathrm{~d}$. These features are unstable under scanning, and it is difficult to acquire higher resolution images to reveal additional details of the structure. However, higher resolution images of the topographically flat areas can be acquired that reveal the lamellar rows on the surface; from the coverage, we infer a thickness of 2-3 ML in these regions. A comparison of such images with larger area images indicates that the topographically bright regions, for example, in Figure $3 \mathrm{~d}$, form at or close to the boundaries between neighboring domains with different orientations. Higher resolution images (see Figure 3f) show that the lamellar rows can deviate from the preferred alignment and show discontinuities and even terminate abruptly in these boundary regions.

As the coverage is further increased to 6.5 ML (Figure $3 \mathrm{~g}-\mathrm{i}$ ) an additional superstructure appears in the form of topographically bright features that run perpendicular to the lamellar rows (in this set of figures, the orientation of the lamellar rows is determined from the high-resolution image in Figure 3i, which is a zoom of the highlighted region in Figure $3 \mathrm{~g})$. Between these bright regions, we observe "trenches" that are topographically dark and also run approximately perpendicular to the lamellar rows (marked by arrows in Figure $3 \mathrm{~h}$ ). This implies that the lamellar rows terminate at positions that are spatially correlated. We speculate that this may be due to strain relief in the growing film, for example, due to a mismatch between the equilibrium alkane spacing and the lattice constant of the $\mathrm{hBN}$.

The lamellar rows and trenches propagate through the growing layer. After the deposition of $10 \mathrm{ML}$, these features may still be resolved although the height profile shows that the surface becomes significantly roughened (Figure $3 j-1$; arrows mark the position of trenches). We do not see further significant changes for thicker layers (Figure $3 \mathrm{~m}-\mathrm{o}$ shows images acquired after the deposition of $30 \mathrm{ML}$ ). Interestingly, the period of the lamellae is preserved for all coverages studied, implying that the substrate directs growth even for these very thick layers.

We have also investigated the effect of postdeposition annealing on the sample morphology (see Figure 4). Figure 4a shows an area on a sample with a coverage of 6.5 ML. The high-contrast region in the center of the image is due to a $\sim 4$ $\mathrm{nm}$ thick hBN terrace, and its structure is independent from the hexacontane deposition. Several different orientational domains are observed, and the boundaries and local orientation of rows are indicated by the turquoise lines and 


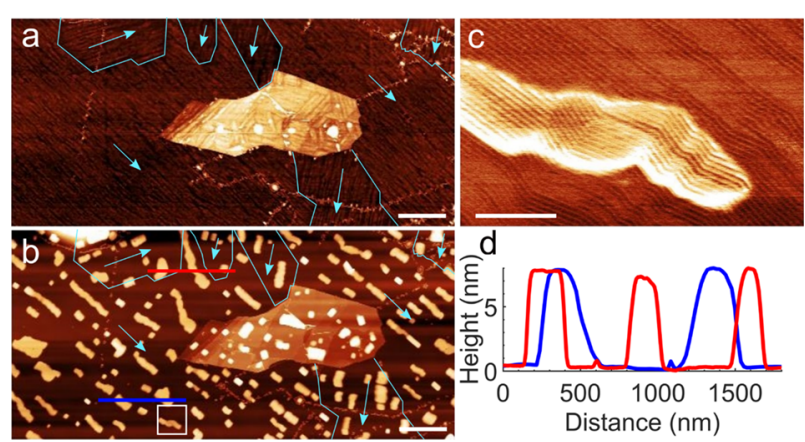

Figure 4. (a, b) Comparison of an hBN area covered in hexacontane before (a) and after (b) heat is applied to the sample. Domain frontiers are indicated in turquoise. (c) AFM phase image showing closely one of the islands formed after heat was applied. The lamellar rows can be resolved on top of the island with the same orientation as in the background space. (d) Profiles running across various islands with paths shown in (b). Scale bars: (a, b) $1 \mu \mathrm{m},(\mathrm{c}) 100 \mathrm{~nm}$.

arrows, respectively. Figure $4 \mathrm{~b}$ shows the same area after the sample was annealed on a hot plate at $80{ }^{\circ} \mathrm{C}$ for $5 \mathrm{~min}$ (under ambient conditions). A series of facetted rectangular islands with heights in the range $6-8 \mathrm{~nm}$ are formed, which are aligned with the orientation of rows prior to annealing. The lamellar rows are still present in the regions of the surface between the islands. The formation of islands is attributed to the agglomeration of hexacontane molecules that are depleted from neighboring multilayer areas in a ripening-type process, which leaves a residual monolayer adsorbed on the hBN surface. Figure $4 \mathrm{c}$ shows an AFM phase image of an island (area marked with a white box in Figure 4b); the corresponding topography image is included in the SI. The lamellar rows are present both on the monolayer-terminated regions between islands and also on the top surface of the islands. Although there is some disorder present, for example, in the fluctuating orientation of the rows, the long axis of the island is approximately parallel to the row direction. Interestingly, these images suggest that in these hexacontane crystallites, the molecules lie parallel to the substrate and the macroscale arrangement consists of stacks of parallel lamellar rows. Furthermore, the orientation of the interfacial hexacontane molecules directly adsorbed on the hBN appears to propagate through the crystallite, to a first approximation, implying an epitaxial relationship.

Finally, we compare the sublimed hexacontane films with those deposited from solution. A sample is immersed in a hexacontane solution ( $10 \mu \mathrm{g}$ of hexacontane $/ \mathrm{mL}$ of toluene) for $1 \mathrm{~min}$, then dried in a nitrogen stream. The molecules form three rotational domains of lamellar structures, with an almost identical period $8.0 \pm 0.2 \mathrm{~nm}$, misaligned by $120^{\circ}$, as shown in Figure 5. The morphology of these films, which have a thickness of $1 \mathrm{ML}$, is very similar to that of the sublimed films, but the size of the domains in this case is much smaller, of the order of $100 \mathrm{~nm}$.

\section{CONCLUSIONS}

Lamellar rows of alkanes are formed on hBN with an internal structure very similar to that observed on a graphite substrate, as confirmed by AFM and molecular mechanics simulations, and in agreement with previous diffraction studies. At higher coverage, the period of the lamellar rows persists and is readily resolved on the surface of multilayers and although some

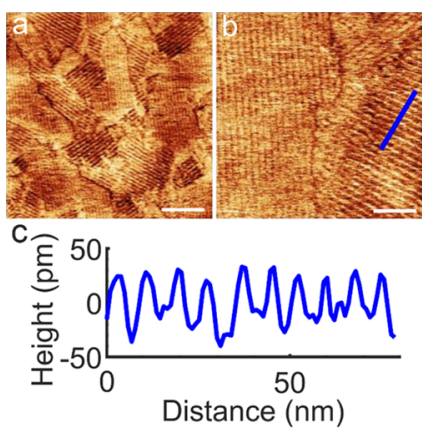

Figure 5. (a, b) AFM images of hexacontane deposited on hBN from solution; (c) height profile along the trajectory marked in (b). Scale bars: (a) $200 \mathrm{~nm}$, (b) $100 \mathrm{~nm}$.

disorder is also evident, for example, in the form of meandering lamellar rows, this implies that growth is directed by the substrate. The hexacontane monolayer provides a very simple example of two-dimensional supramolecular organization. Our results therefore strongly suggest that chain-chain interactions can direct the formation of self-assembled molecular arrays on $\mathrm{hBN}$ in a manner analogous to the highly successful approach to supramolecular organization of complex structures on graphite. ${ }^{17-20}$ In particular, the possible use of $\mathrm{hBN}$ raises the possibility of exploring optical properties of such structures that are difficult to access on conducting surfaces, and we believe that this combination of an insulating substrate and van der Waals mediated molecular organization represents a promising route for the future investigation of the science and technology of surface-stabilized supramolecular arrays.

\section{ASSOCIATED CONTENT}

\section{Supporting Information}

The Supporting Information is available free of charge on the ACS Publications website at DOI: 10.1021/acs.jpcc.8b10167.

Details of numerical procedures; additional AFM images; and examples of AFM-induced modification (PDF)

The original data files including raw AFM images may be found at http://dx.doi.org/10.17639/nott.376.

\section{AUTHOR INFORMATION}

\section{Corresponding Author}

*E-mail: peter.beton@nottingham.ac.uk.

ORCID

Elena Besley: 0000-0002-9910-7603

Peter H. Beton: 0000-0002-2120-8033

Notes

The authors declare no competing financial interest.

\section{ACKNOWLEDGMENTS}

This work was supported by the Engineering and Physical Sciences Research Council [grant numbers EP/N033906/1, EP/P019080/1]; the Leverhulme Trust [grant number RPG2016-104]. K.W. and T.T. acknowledge support from the Elemental Strategy Initiative conducted by the MEXT, Japan and the CREST (JPMJCR15F3), JST.

\section{REFERENCES}

(1) Grant, J. T.; Carrero, C. A.; Goeltl, F.; Venegas, J.; Mueller, P.; Burt, S. P.; Specht, S. E.; McDermott, W. P.; Chieregato, A.; 
Hermans, I. Selective Oxidative Dehydrogenation of Propane to Propene Using Boron Nitride Catalysts. Science 2016, 354, 15701573.

(2) Altwasser, S.; Welker, C.; Traa, Y.; Weitkamp, J. Catalytic Cracking of N-Octane on Small-Pore Zeolites. Microporous Mesoporous Mater. 2005, 83, 345-356.

(3) Sivebaek, I. M.; Samoilov, V. N.; Persson, B. N. J. Squeezing Molecular Thin Alkane Lubrication Films between Curved Solid Surfaces with Long-Range Elasticity: Layering Transitions and Wear. J. Chem. Phys. 2003, 119, 2314-2321.

(4) Collins, G.; Fleming, P.; Barth, S.; O’Dwyer, C.; Boland, J. J.; Morris, M. A.; Holmes, J. D. Alkane and Alkanethiol Passivation of Halogenated Ge Nanowires. Chem. Mater. 2010, 22, 6370-6377.

(5) Sattler, J. J. H. B.; Ruiz-Martinez, J.; Santillan-Jimenez, E.; Weckhuysen, B. M. Catalytic Dehydrogenation of Light Alkanes on Metals and Metal Oxides. Chem. Rev. 2014, 114, 10613-10653.

(6) Morikawa, Y.; Ishii, H.; Seki, K. Theoretical Study of N-Alkane Adsorption on Metal Surfaces. Phys. Rev. B 2004, 69, No. 041403.

(7) Weaver, J. The Adsorption and Reaction of Low Molecular Weight Alkanes on Metallic Single Crystal Surfaces. Surf. Sci. Rep. 2003, 50, 107-199.

(8) Ilan, B.; Florio, G. M.; Hybertsen, M. S.; Berne, B. J.; Flynn, G. W. Scanning Tunneling Microscopy Images of Alkane Derivatives on Graphite: Role of Electronic Effects. Nano Lett. 2008, 8, 3160-3165.

(9) Yang, T.; Berber, S.; Liu, J.-F.; Miller, G. P.; Tománek, D. SelfAssembly of Long Chain Alkanes and Their Derivatives on Graphite. J. Chem. Phys. 2008, 128, No. 124709.

(10) Hibino, M. Adsorption Behaviors of Mixed Monolayers of N -Alkanes at the Liquid-Solid Interface. Langmuir 2016, 32, 47054709.

(11) Gosvami, N. N.; O'Shea, S. J. Nanoscale Trapping and Squeeze-Out of Confined Alkane Monolayers. Langmuir 2015, 31, 12960-12967.

(12) Svatek, S. A.; Scott, O. R.; Rivett, J. P. H.; Wright, K.; Baldoni, M.; Bichoutskaia, E.; Taniguchi, T.; Watanabe, K.; Marsden, A. J.; Wilson, N. R.; et al. Adsorbate-Induced Curvature and Stiffening of Graphene. Nano Lett. 2015, 15, 159-164.

(13) Rabe, J. P.; Buchholz, S. Commensurability and Mobility in Two-Dimensional Molecular Patterns on Graphite. Science 1991, 253, 424-427.

(14) McGonigal, G. C.; Bernhardt, R. H.; Thomson, D. J. Imaging Alkane Layers at the Liquid/graphite Interface with the Scanning Tunneling Microscope. Appl. Phys. Lett. 1990, 57, 28-30.

(15) Cyr, D. M.; Venkataraman, B.; Flynn, G. W. STM Investigations of Organic Molecules Physisorbed at the LiquidSolid Interface. Chem. Mater. 1996, 8, 1600-1615.

(16) Baker, R. T.; Mougous, J. D.; Brackley, A.; Patrick, D. L. Competitive Adsorption, Phase Segregation, and Molecular Motion at a Solid-Liquid Interface Studied by Scanning Tunneling Microscopy. Langmuir 1999, 15, 4884-4891.

(17) Leysen, P.; Teyssandier, J.; De Feyter, S.; Koeckelberghs, G. Controlled Synthesis of a Helical Conjugated Polythiophene. Macromolecules 2018, 51, 3504-3514.

(18) Lee, S.-L.; Fang, Y.; Velpula, G.; Cometto, F. P.; Lingenfelder, M.; Müllen, K.; Mali, K. S.; De Feyter, S. Reversible Local and Global Switching in Multicomponent Supramolecular Networks: Controlled Guest Release and Capture at the Solution/Solid Interface. ACS Nano 2015, 9, 11608-11617.

(19) Goronzy, D. P.; Ebrahimi, M.; Rosei, F.; Arramel; Fang, Y.; De Feyter, S.; Tait, S. L.; Wang, C.; Beton, P. H.; Wee, A. T. S.; et al. Supramolecular Assemblies on Surfaces: Nanopatterning, Functionality, and Reactivity. ACS Nano 2018, 12, 7445-7481.

(20) Teyssandier, J.; Feyter, S.; Mali, K. S. Host-guest Chemistry in Two-Dimensional Supramolecular Networks. Chem. Commun. 2016, 52, 11465-11487.

(21) Geim, A. K.; Grigorieva, I. V. Van Der Waals Heterostructures. Nature 2013, 499, No. 419.

(22) Cho, Y. J.; Summerfield, A.; Davies, A.; Cheng, T. S.; Smith, E. F.; Mellor, C. J.; Khlobystov, A. N.; Foxon, C. T.; Eaves, L.; Beton, P.
H.; et al. Hexagonal Boron Nitride Tunnel Barriers Grown on Graphite by High Temperature Molecular Beam Epitaxy. Sci. Rep. 2016, 6, No. 34474.

(23) Dean, C. R.; Young, A. F.; Meric, I.; Lee, C.; Wang, L.; Sorgenfrei, S.; Watanabe, K.; Taniguchi, T.; Kim, P.; Shepard, K. L.; et al. Boron Nitride Substrates for High-Quality Graphene Electronics. Nat. Nanotechnol. 2010, 5, 722-726.

(24) Jain, N.; Bansal, T.; Durcan, C.; Yu, B. Graphene-Based Interconnects on Hexagonal Boron Nitride Substrate. IEEE Electron Device Lett. 2012, 33, 925-927.

(25) Kumar, A.; Banerjee, K.; Liljeroth, P. Molecular Assembly on Two-Dimensional Materials. Nanotechnology 2017, 28, No. 082001.

(26) Schulz, F.; Drost, R.; Hämäläinen, S. K.; Liljeroth, P. Templated Self-Assembly and Local Doping of Molecules on Epitaxial Hexagonal Boron Nitride. ACS Nano 2013, 7, 11121-11128.

(27) Berner, S.; Corso, M.; Widmer, R.; Groening, O.; Laskowski, R.; Blaha, P.; Schwarz, K.; Goriachko, A.; Over, H.; Gsell, S.; et al. Boron Nitride Nanomesh: Functionality from a Corrugated Monolayer. Angew. Chem., Int. Ed. 2007, 46, 5115-5119.

(28) Pollard, A. J.; Perkins, E. W.; Smith, N. A.; Saywell, A.; Goretzki, G.; Phillips, A. G.; Argent, S. P.; Sachdev, H.; Müller, F.; Hüfner, S.; et al. Supramolecular Assemblies Formed on an Epitaxial Graphene Superstructure. Angew. Chem., Int. Ed. 2010, 49, 17941799.

(29) Korolkov, V. V.; Svatek, S. A.; Summerfield, A.; Kerfoot, J.; Yang, L.; Taniguchi, T.; Watanabe, K.; Champness, N. R.; Besley, N. A.; Beton, P. H. Van Der Waals-Induced Chromatic Shifts in Hydrogen-Bonded Two-Dimensional Porphyrin Arrays on Boron Nitride. ACS Nano 2015, 9, 10347-10355.

(30) Matković, A.; Genser, J.; Lüftner, D.; Kratzer, M.; Gajić, R.; Puschnig, P.; Teichert, C. Epitaxy of Highly Ordered Organic Semiconductor Crystallite Networks Supported by Hexagonal Boron Nitride. Sci. Rep. 2016, 6, No. 38519.

(31) Zhang, Y.; Qiao, J.; Gao, S.; Hu, F.; He, D.; Wu, B.; Yang, Z.; $\mathrm{Xu}, \mathrm{B}$.; Li, Y.; Shi, Y.; et al. Probing Carrier Transport and StructureProperty Relationship of Highly Ordered Organic Semiconductors at the Two-Dimensional Limit. Phys. Rev. Lett. 2016, 116, No. 16602.

(32) Arnold, T.; Forster, M.; Athanasiou Fragkoulis, A.; Parker, J. E. Structure of Normal-Alkanes Adsorbed on Hexagonal-Boron Nitride. J. Phys. Chem. C 2014, 118, 2418-2428.

(33) Forster, M.; Parker, J. E.; Inaba, A.; Murray, C. A.; Strange, N. A.; Larese, J. Z.; Arnold, T. The Mixing Behavior of Alkanes Adsorbed on Hexagonal Boron Nitride. J. Phys. Chem. C 2016, 120, 2579625805.

(34) Korolkov, V. V.; Svatek, S. A.; Allen, S.; Roberts, C. J.; Tendler, S. J. B.; Taniguchi, T.; Watanabe, K.; Champness, N. R.; Beton, P. H. Bimolecular Porous Supramolecular Networks Deposited from Solution on Layered Materials: Graphite, Boron Nitride and Molybdenum Disulphide. Chem. Commun. 2014, 50, 8882-8885.

(35) Korolkov, V. V.; Baldoni, M.; Watanabe, K.; Taniguchi, T.; Besley, E.; Beton, P. H. Supramolecular Heterostructures Formed by Sequential Epitaxial Deposition of Two-Dimensional HydrogenBonded Arrays. Nat. Chem. 2017, 9, 1191-1197.

(36) Taniguchi, T.; Watanabe, K. Synthesis of High-Purity Boron Nitride Single Crystals under High Pressure by Using Ba-BN Solvent. J. Cryst. Growth 2007, 303, 525-529.

(37) Holzwarth, A.; Leporatti, S.; Riegler, H. Molecular Ordering and Domain Morphology of Molecularly Thin Triacontane Films at SiO 2/air Interfaces. Europhys. Lett. 2000, 52, 653-659.

(38) Korolkov, V. V.; Timokhin, I. G.; Haubrichs, R.; Smith, E. F.; Yang, L.; Yang, S.; Champness, N. R.; Schröder, M.; Beton, P. H. Supramolecular Networks Stabilise and Functionalise Black Phosphorus. Nat. Commun. 2017, 8, No. 1385.

(39) Groszek, A. J. Selective Adsorption at Graphite/Hydrocarbon Interfaces. Proc. R. Soc. A 1970, 314, 473-498.

(40) Plimpton, S. Fast Parallel Algorithms for Short-Range Molecular Dynamics. J. Comput. Phys. 1995, 117, 1-19. 
(41) Siu, S. W. I.; Pluhackova, K.; Böckmann, R. A. Optimization of the OPLS-AA Force Field for Long Hydrocarbons. J. Chem. Theory Comput. 2012, 8, 1459-1470.

(42) Lee, J. H. A Study on a Boron-Nitride Nanotube as a Gigahertz Oscillator. J. Korean Phys. Soc. 2006, 49, 172-176. 